\title{
Hardware Performance Optimization and Evaluation of SM3 Hash Algorithm on FPGA
}

\author{
Yuan Ma ${ }^{1,2, \star}$, Luning Xia ${ }^{1}$, Jingqiang Lin ${ }^{1}$, Jiwu Jing ${ }^{1}$, \\ Zongbin $\mathrm{Liu}^{1}$, and Xingjie $\mathrm{Yu}^{1,2}$ \\ 1 State Key Laboratory of Information Security, \\ Institute of Information Engineering, CAS, Beijing, China \\ 2 Graduate University of Chinese Academy of Sciences, Beijing, China \\ \{yma, halk, linjq, jing, zbliu, xjyu\}@lois.cn
}

\begin{abstract}
Hash algorithms are widely used for data integrity and authenticity. Chinese government recently published a standard hash algorithm, SM3, which is highly recommended for commercial applications. However, little research of SM3 implementation has been published. We find that the existing optimization techniques cannot be adopted to SM3 efficiently, due to the complex computation and strong data dependency. In this paper, we present our novel optimization techniques: shift initialization and SRL-based implementation. Based on the techniques, we propose two architectures: compact design and high-throughput design, both of which significantly improve the performance on FPGA. As far as we know, our work is the first one to evaluate SM3 hardware performance. The evaluation result suggests that SM3 with low area and high efficiency is suitable for hardware implementations, especially for those resource-limited platforms.
\end{abstract}

Keywords: SM3, hash algorithm, FPGA, optimization, hardware performance evaluation.

\section{Introduction}

At present, the performance in hardware has been demonstrated to be an important factor in the evaluation of cryptographic algorithms. The ASIC (ApplicationSpecific Integrated Circuit) and FPGA (Field-Programmable Gate Array) are two common hardware devices for cryptographic implementations. FPGA implementation has become more and more popular recently since it is reconfigurable and relatively flexible.

Hash algorithms that compute a fixed-length message digest from arbitrary length messages are widely used for many purposes in information security. SM3 hash algorithm [1] was published by Chinese Commercial Cryptography Administration Office in December, 2010. As the only standard hash algorithm of China

\footnotetext{
* This work was supported by National Natural Science Foundation of China (Grant No. 70890084/G021102, 61003274 and 61003273) and Strategy Pilot Project of Chinese Academy of Sciences, Sub-Project XDA06010702.
} 
and the replacement of other hash algorithms, SM3 is being integrated into most commercial security products in China.

Up to now, there are no results for hardware implementation of SM3 in literature. As far as we know, our work is the first one to evaluate SM3 hardware performance. Although SM3 can be implemented in a similar way as SHA-1 and SHA-256 which have a similar structure, the common optimization techniques cannot be efficiently applied for SM3 implementations, due to the complex computation and strong data-dependency in the iteration. Furthermore, when SM3 is implemented in FPGA without specific optimizations, we find that the use of registers and LUTs (Look-Up Tables) is not balanced. Much more registers are used than LUTs. So the number of registers will determine the number of occupied slices. Apparently, the imbalance increases the area and reduces the resource utilization.

Our goal is to propose new optimization techniques for SM3 implementation, either to minimize the area or to maximize the throughput. The paper makes two contributions as follows.

1. We propose a very compact architecture for processing one message.

Compared with the "standard" (defined in Section 5) SM3 implementation, the compact one only occupies $60 \%$ area with no degradation of throughput. The compact architecture is based on our novel optimization techniques - shift initialization and SRL (Shift Register LUT) based implementation, which are based on the following observations.

- There is a unique characteristic in SM3 algorithm. For processing one data block, the message expansion takes 68 clock cycles, while the iteration takes 64 rounds. It indicates that in the first four cycles, the iteration module is idle.

- Some area-saving optimization can be done by utilizing the idle four cycles, which means trading time for space.

- The initialization circuit occupies substantial registers and LUTs. Instead of initializing in one cycle, the circuit can complete the process in the additional four clock cycles.

- SRL is helpful to balance registers and LUTs in FPGA.

2. We propose a high-throughput architecture for processing two concurrent messages alternatingly.

Our optimization techniques are more efficient for the two-message architecture. Compared with the stand implementation, the high-throughput one improves the throughput by $69 \%$, and also saves $17 \%$ area. Compared with the compact implementation, the high-throughput one also achieves a $68 \%$ throughput improvement with only a $40 \%$ area increase, resulting in that the ratio of throughput to area increases by $20 \%$. The high-throughput architecture is inspired by the following observations.

- By inserting registers in the critical path, the strong data dependency can be avoided for processing two independent message. And the throughput will significantly increase. 
- The two messages can share the same computation unit for compression and expansion in an alternative form. Thus, the LUT resources are reused.

- However, the registers for variable storage have to be doubled for processing two messages. Fortunately, the shift initialization structure and SRL-based implementation can provide a good means to minimize the register consumption.

The rest of this paper is organized as follows. Section 2 presents the SM3 hash algorithm. Section 3 analyzes the critical path and describes our optimization techniques and the compact architecture. Section 4 proposes the high-throughput architecture. Section 5 defines the standard implementation and presents the implementation and comparison results. Section 6 presents related works. Section 7 concludes the paper.

\section{Preliminary: SM3 Hash Algorithm}

The SM3 hash algorithm processes a message of length $l\left(l<2^{64}\right)$ bits to produce a final digest message of 256 bits after padding and compressing. The message, which is composed of multiple blocks of 512 bits each after padding, is expanded and fed to the 64 cycles of the compression function in words of 32 bits each.

\subsection{Message Padding and Parsing}

The binary message of length $l$ to be processed is appended with a '1' and padded with zeros of length $k$ until $(l+k+1) \equiv 448 \bmod 512$. And the resultant padded message is parsed into $N 512$-bit blocks, denoted $B(1), B(2), \ldots, B(N)$. These $B(i)$ message blocks are fed individually to the message expander.

\section{$2.2 \quad$ Message Expansion}

The functions in the SM3 algorithm operate on 32-bit words, so each 512-bit block $B(i)$ from the padding stage is viewed as 1632 -bit blocks denoted $B_{j}{ }^{(i)}, 0 \leq$ $j \leq 15$. The message expander takes each $B(i)$ and expands it to form 6832 -bit blocks $W_{j}$ and 6432 -bit blocks $W_{j}{ }^{\prime}$, according to the equations:

$$
P_{1}(X)=X \oplus(X \lll 15) \oplus(X \lll 23)
$$

$$
W_{j}=\left\{\begin{array}{lr}
B_{j}{ }^{(i)}, & 0 \leq j \leq 15 ; \\
P_{1}\left(W_{j-16} \oplus W_{j-9} \oplus\left(W_{j-3} \lll 15\right)\right) \oplus\left(W_{j-13} \lll 7\right) \oplus W_{j-6}, & 16 \leq j \leq 67 .
\end{array}\right.
$$

$$
W_{j}{ }^{\prime}=W_{j} \oplus W_{j+4}
$$

where $x \lll n$ denotes a circular rotation of $x$ by $n$ positions to the left. All additions in the SM3 algorithm are modulo $2^{32}$. 


\subsection{Message Compression}

The $W_{j}$ and $W_{j}{ }^{\prime}$ words from the message expansion stage are then passed to the SM3 compression function. The function utilizes 8 32-bit working variables labeled $A, B, C, D, E, F, G, H$, which are initialized to predefined values $V_{0}{ }^{(0)}, \ldots, V_{7}{ }^{(0)}$ at the start of each call to the hash function. Sixty-four iterations of the compression function are then performed, given by:

$$
\begin{aligned}
& S S 1=\left((A \lll 12)+E+\left(T_{j} \lll j\right)\right) \lll 7 \\
& S S 2=S S 1 \oplus(A \lll 12) \\
& T T 1=F F_{j}(A, B, C)+D+S S 2+W_{j}^{\prime} \\
& T T 2=G G_{j}(E, F, G)+H+S S 1+W_{j} \\
& D=C \\
& B=A
\end{aligned} \quad \begin{array}{ll}
C=B \lll 9 \\
H=G & A=T T 1 \\
F=E & E=F \lll 19 \\
& E=P_{0}(T T 2)
\end{array}
$$

where

$$
T_{j}=\left\{\begin{array}{l}
79 c c 4519,0 \leq j \leq 15 \\
7 a 879 d 8 a, 16 \leq j \leq 63
\end{array}\right.
$$

and the functions denoted $F F_{j}, G G_{j}$ and $P_{0}$ are given by:

$$
\begin{gathered}
F F_{j}(X, Y, Z)=\left\{\begin{array}{l}
X \oplus Y \oplus Z, \\
(X \wedge Y) \vee(X \wedge Z) \vee(Y \wedge Z), 16 \leq j \leq 63 .
\end{array}\right. \\
G G_{j}(X, Y, Z)=\left\{\begin{array}{l}
X \oplus Y \oplus Z, \\
(X \wedge Y) \vee(\neg X \wedge Z) \vee(Y \wedge Z), 16 \leq j \leq 63 .
\end{array}\right. \\
P_{0}(X)=X \oplus(X \lll 9) \oplus(X \lll 17)
\end{gathered}
$$

where $\oplus$ represents the bitwise $X O R$ operation, $\vee$ the bitwise $O R$ operation, $\wedge$ the bitwise $A N D$ operation and $\neg$ the bitwise NOT operation.

After 64 iterations of the compression function, an intermediate hash value $V^{(i)}$ is calculated:

$$
V_{0}^{(i)}=A \oplus V_{0}^{(i-1)}, V_{1}^{(i)}=B \oplus V_{1}^{(i-1)}, \ldots, V_{7}^{(i)}=H \oplus V_{1}^{(i-1)}
$$

The SM3 compression algorithm then repeats and begins processing another 512bit block from the message padder. After all $N$ data blocks have been processed, the final 256-bit output, $V^{(N)}$, is formed by concatenating the final hash values:

$$
V^{(N)}=V_{0}^{(N)} V_{1}^{(N)} \ldots V_{7}^{(N)}
$$




\section{Proposed Compact Architecture}

In this section, based on the analysis of the critical path, we find that the fixed critical path limits the maximum frequency and the throughput. Then we turn to minimize the area to improve the ratio of throughput to area. We propose a shift initialization structure to reuse hardware resources, and the SRL-based implementation to balance registers and LUTs. Based on them, the compact architecture is proposed.

\subsection{Critical Path}

The critical path refers to the one which creates the longest delay, and it limits the maximum working frequencies of systems. From Equations (4), it can be observed that the SM3 round computation is oriented towards the $A$ and $E$ value calculations. The remaining values do note require any computation, aside from the rotations of $B$ and $F$. The values of $\mathrm{A}$ and $\mathrm{E}$ are both computed from $S S 1$, which depends on the previous values of $A$ and $E$, as depicted in Equations (11)- (13).

$$
\begin{aligned}
S S 1 & =\left(A \lll 12+E+T_{j} \lll j\right) \lll 7 \\
A & =F F_{j}(A, B, C)+D+W_{j}{ }^{\prime}+S S 1 \oplus(A \lll 12) \\
E & =P_{0}\left(G G_{j}(E, F, G)+H+W_{j}+S S 1\right)
\end{aligned}
$$

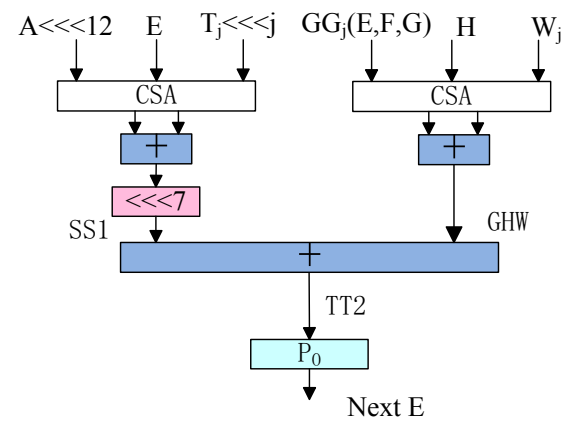

Fig. 1. The critical path of SM3

Note that the rotation operation does not require additional resources in hardware, and $S S 1 \oplus(A \lll 12)$ can be synthesized with the addition to one-stage LUTs in FPGA. Thus, Equation (13), E path, is longer than Equation (12) by a delay of one-stage LUTs. The additional LUTs are used to implement function $P_{0}$. For $E$ path, the computations of $S S 1$ and $G G_{j}+H+W_{j}$ that are both the addition of three numbers can be processed simultaneously. The structure of the critical path of SM3 algorithm is depicted in Figure 1. 
The additions of three numbers are optimized by CSA (Carry Save Adder), which separates the sum and carry paths and minimizes the delay caused by traditional carry propagation. The rotation $\lll 7$ sets up a major barrier for optimizing the critical path, because it isolates the additions of $S S 1$ from others. Thus $S S 1$ has to be computed separately. Pre-computing $H+W_{j}$ is useless for shortening the critical path, as the computation of $S S 1$ is still in the critical path. Further more, both $A$ and $E$ depend on their values of the previous round. Thus, the critical path is almost fixed, due to the strong data dependency. Thus, the throughput is hardly improved due to such a critical path. For the SM3 implementation, in order to improve the ratio of throughput to area, our optimization goal is to minimize the resource consumption.

\subsection{Message Expansion}

The input data block expansion described in Equations (1)-(3), can be implemented with registers and XOR operations. The output value is selected between the original data block, for the first 16 rounds, and the computed values, for the remaining rounds. Figure 2 depicts the implemented structure. In order to eliminate this expansion computation from the critical path, both $W$ and $W^{\prime}$ are the outputs of registers.

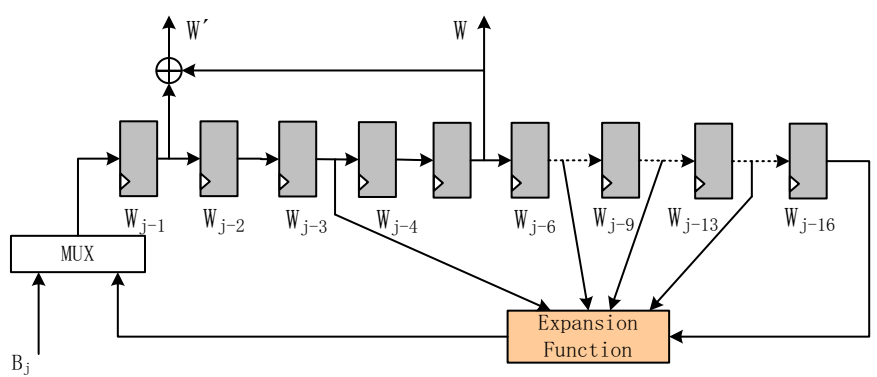

Fig. 2. The SM3 message expansion structure

SRL-Based Implementation. In the register-based structure, sixteen 32-bit width registers are needed to implement the expander. It can be observed that most of them are only used to temporarily store data, so we use SRLs to replace the shift register for area saving.

In FPGA, LUTs can also be configured as 16-bit shift registers, as shown in Figure 3. One SRL can implement a 1-bit wide, 16-bit long shift register. Thus, SRL used to reduce registers provides a way to balance registers and LUTs. The input $C E$ is used to control data shift, and $A d d r$ is used to change the length of the shift register dynamically. In addition, the SRL contents can be initialized by designers.

In SM3 message expansion, the registers used to create the temporal delay of $W_{j}$ can be replaced by SRLs, as shown in Figure 4. where SRL-x represents a 
SRL

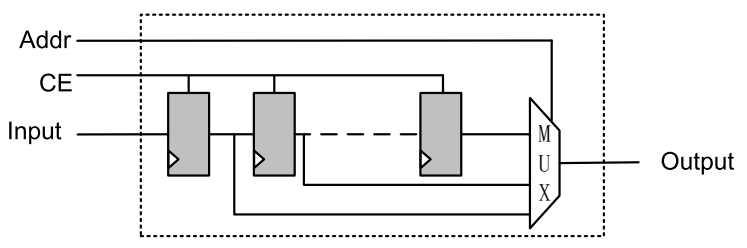

Fig. 3. The Shift Register LUT structure

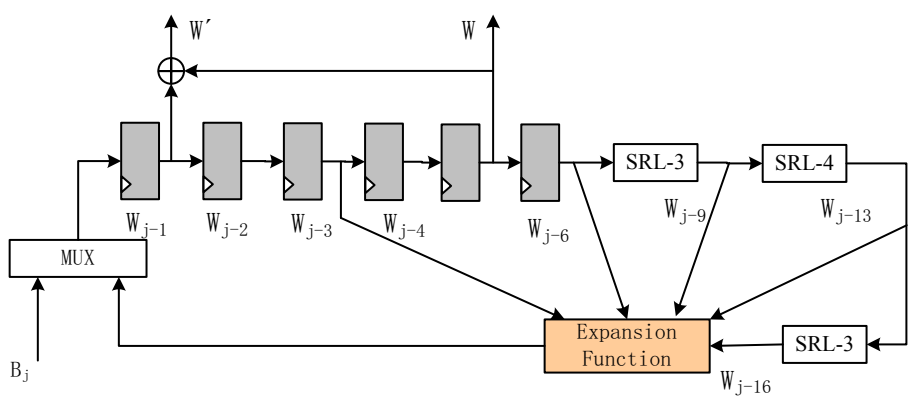

Fig. 4. SRL-based message expansion structure

32-bit wide, $\mathrm{x}$-bit long shift register. Only $6 \times 32$ registers and $3 \times 32 \mathrm{SRLs}$ are used to implement the 32-bit 16-length shift registers.

It should be noticed that $W_{j}{ }^{\prime}=W_{j} \oplus W_{j+4}$ implying that the first $W_{0}{ }^{\prime}$ will not be obtained until $j=4$. Due to this fact, the compressor starts to iterate 64 rounds after its input $W_{0}^{\prime}$ is generated at the 4 th clock round. Therefore, the SM3 module takes 68 cycles to process one 512-bit chunk of data with the additional 4 clock cycles for initializing $W_{j}{ }^{\prime}$. In fact, our optimization techniques are inspired by the additional four cycles.

\subsection{SIS-Based Compact Architecture}

Shift Initialization Structure (SIS). Before every 64-round iteration, variables $A$ to $H$ should be initialized using intermediate hash values $V_{0}$ to $V_{7}$. And after the computation of a given data block, the finial values of internal variables $A$ to $H$ are XORed to the current intermediate hash values $V_{0}$ to $V_{7}$. Especially, for the first message block, $V_{0}$ to $V_{7}$ are initialized by constants $I V$. If these operations were implemented in a straightforward manner, eight multiplexers and eight XOR gates would be required. In this section, we propose a shift initialization structure that can efficiently completes the initialization and XOR operation with a low area.

When the message expander is initializing $W_{j}{ }^{\prime}$ in the first four clock cycles, the compressor is idle. Therefore, instead of completing XOR in one clock cycle, we use shifted registers to operate that in the four cycles. The SIS is based on 
Equations (14), as presented in the most right part of Figure 5. Furthermore, using shift registers has another advantage, allowing us to replace the 256 registers $V_{0}$ to $V_{7}$ with only 64 SRLs.

$$
\begin{aligned}
& \text { for } j=0 \text { to } j=3 \text { do } \\
& \{D, C, B, A\}=\left\{C, B, A, V_{3} \oplus D\right\} \\
& \{H, G, F, E\}=\left\{G, F, E, V_{7} \oplus H\right\} \\
& \text { endfor } \\
& \text { for } j=0 \text { to } j=4 \text { do } \\
& \left\{V_{3}, V_{2}, V_{1}, V_{0}\right\}=\left\{V_{2}, V_{1}, V_{0}, A\right\} \\
& \left\{V_{7}, V_{6}, V_{5}, V_{4}\right\}=\left\{V_{6}, V_{5}, V_{4}, E\right\}
\end{aligned}
$$

When configured, the SRLs for $V_{0}$ to $V_{7}$ are initialized using the constant $I V$. For the first message block, the values of $V_{0}$ to $V_{7}$ are derived from $I V$, and the values of $A$ to $H$ are set to zero. After the shift initialization, both $V_{0}$ to $V_{7}$ and $A$ to $H$ equal the value of XOR zero to $I V$, i.e. $I V$, meaning that the initialization properly complete.

Proposed Compact Architecture. Based on SIS, we propose a very compact SM3 architecture, depicted in Figure 5 which is approximately symmetrical. Three CSAs are used to reduce the path delay, followed by five 32-bit adders that can be implemented conveniently by Carry Propagate Adders (CPAs) in FPGAs.

The shift initialization, as shown in the most right part of Figure [5, also allows us to remove many multiplexers. For variables $B, D, F$ and $H$ the multiplexers are saved, because whenever in the first four cycles or in the next 64-round iterations the assignments for them are not changing. But for $C$ and $G$, the multiplexers are needed as the rotation operation exists. Notice that the XOR gate and multiplexer used for $E$ in the right part of Figure 5 don't increase the resource consumption and the critical path, because they can be synthesized to one-stage LUTs of six inputs with the function $P_{0}$. That is why we do not use the output of XOR gates to update $V_{0}$ and $V_{4}$, as the XOR gates can be synthesized with the multiplexers.

In this architecture, only 160 LUTs, that divided into 5 groups of 32 , are required for the whole round implementation on the basis of the iteration structure. Two groups are used for the two multiplexers of $C$ and $G$, two for the SRL-4's, and the last one for the multiplexer and XOR gate of $A$.

Efficient Shift Output. We use the SRL-based implementation for a efficient reading of the final hash values. Addr, the input of SRL which acts as a RAM, can be used to dynamically change the length of shift register. That means we can control $A d d r$ to obtain any register value in the SRL as long as we know the right address of that register. Thus, when $C E$ is low, we successively increase $A d d r$ from 2-bit 00 to 2-bit 11 to obtain $V_{0}$ to $V_{3}$ and $V_{4}$ to $V_{7}$ sequentially. 


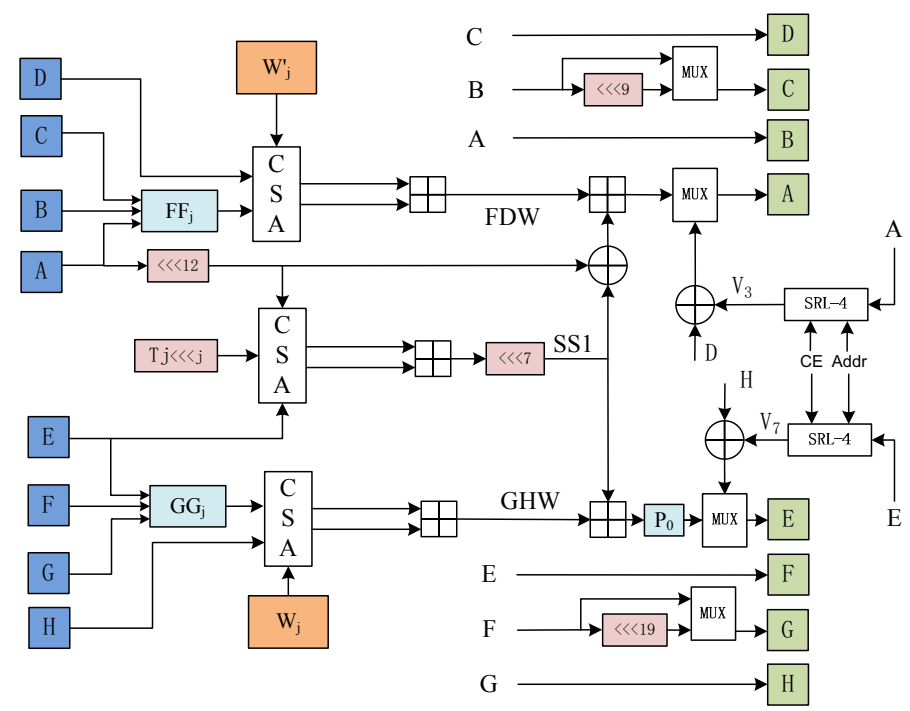

Fig. 5. The proposed compact SM3 architecture

\section{Proposed High-Throughput Architecture}

In this section, in order to further improve resource utilization, we propose a high-throughput architecture that can processes two independent messages alternatingly. That is motivated by the long and symmetrical critical path in SM3. We split the critical path in half by inserting registers to increase the maximum frequency and throughput. Meanwhile, the iteration and expansion LUT circuits are reused in our high-throughput architecture. Furthermore, the SRL optimization technique can be used more efficiently for registers saving in the architecture, yielding a higher throughput/area.

\subsection{High-Throughput Architecture}

We propose a high-throughput architecture that processes two independent messages alternatingly, as shown in Figure 6 where $F D W, S S 1$ and $G H W$ are the inserted registers. The computation of these register variables are presented in Equations (15), which are all three-number additions, labeled Stage 1 in Figure 6. The additions are implemented by CSA that consists of one-stage LUT and one 32-bit CPA. For the path after the registers labeled Stage 2 in Figure 6 . either for $A$ or $E$, the calculating path is also one 32-bit CPA and one-stage LUT. Thus, The critical path is shorten by half.

$$
\begin{aligned}
F D W & =F F_{j}(A, B, C)+D+W_{j}{ }^{\prime} \\
S S 1 & =\left(A \lll 12+E+T_{j} \lll j\right) \lll 7 \\
G H W & =G G_{j}(E, F, G)+H+W_{j}
\end{aligned}
$$


For other variables $B$ to $D$ and $F$ to $H$, one more stage of registers, named $A_{r}$ to $C_{r}$ and $E_{r}$ to $G_{r}$ in Figure 6 , are required to ensure that these variables are updated at the same round with $A$ and $E$.

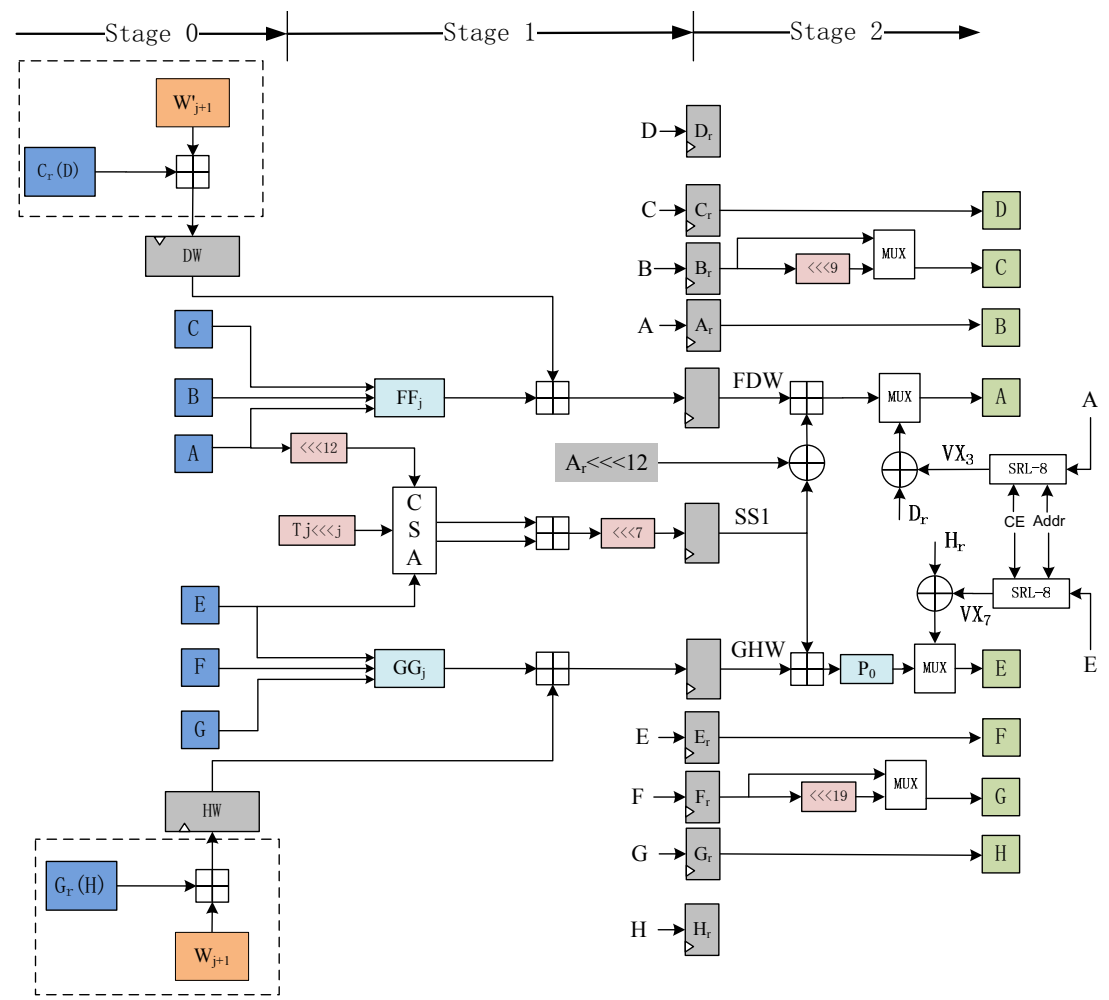

Fig. 6. The high-throughput round architecture of SM3

SIS-Based Implementation. SIS can be embedded efficiently in the architecture, as depicted in the most right part of Figure [6. In the architecture, the shift initialization for two messages runs according to Equations (16). The initialization process here is similar to that in the compact architecture, except for processing two messages alternatingly.

$$
\text { for } \begin{aligned}
j=0 \text { to } j=7 \text { do } \\
\left\{D_{r}, D, C_{r}, \ldots, A_{r}, A\right\}=\left\{D, C_{r}, C, \ldots, A, V X_{3} \oplus D_{r}\right\} \\
\left\{H_{r}, H, G_{r}, \ldots, E_{r}, E\right\}=\left\{H, G_{r}, G, \ldots, E, V X_{7} \oplus H_{r}\right\}
\end{aligned}
$$

endfor

$$
\text { for } \begin{aligned}
j=0 \text { to } j=8 \text { do } \\
\quad\left\{V X_{3}, V Y_{3}, V X_{2}, \ldots, V X_{0}, V Y_{0}\right\}=\left\{V Y_{3}, V X_{2}, V Y_{2}, \ldots, V Y_{0}, A\right\} \\
\left\{V X_{7}, V Y_{7}, V X_{6}, \ldots, V X_{4}, V Y_{4}\right\}=\left\{V Y_{7}, V X_{6}, V Y_{6}, \ldots, V Y_{4}, E\right\}
\end{aligned}
$$

endfor 
Instead of two SRL-4's in the compact architecture, two SRL-8's are needed to store eight intermediate values $V X$ and $V Y$ for $X$ and $Y$ alternatingly. More precisely, the first SRL- 8 stores intermediate values in order of $\left\{V X_{3}, \ldots, V Y_{0}\right\}$, and the second SRL- $8\left\{V X_{7}, \ldots, V Y_{4}\right\}$. Notice that this change does not increase LUT consumption, because SRL-4 and SRL-8, the difference of which is the length of shift register, are both implemented by 32 LUTs. Furthermore, $D_{r}$ and $H_{r}$ used to temporarily store $D$ and $H$ are needed for executing XOR with $V X_{3}$ and $\mathrm{VX}_{7}$ in Figure 6 .

Adding Additional Pipeline. We add additional pipeline named Stage 0 for keeping the critical path as expected, as labeled in the two dotted frames of Figure 6. One-stage LUTs are not enough to implement the CSA for $F D W$, because the total number of input variables is seven, as shown Equations (15) where $W_{j}^{\prime}=W_{j} \oplus W_{j+4}$. Thus we compute the additions $D+W_{j}^{\prime}$ and $H+W_{j}$ in the previous round. Stage 0 pre-computes the additions using $C_{r}+W_{j+1}^{\prime}$ and $G_{r}+W_{j+1}$ of the previous round.

With this pipeline, an additional clock cycle is required for each block. The additional cycle, however, can be hidden in the initialization of $A$ to $H$. Therefore, each 512-bit chunk of the two messages can be processed within 136 clock cycles, 68 cycles for each message.

\subsection{Message Expansion}

Similar to the message expansion in the compact architecture, the expander for two alternative messages are implemented using SRL, as shown in Figure 7 . Only $6 \times 32$ registers and $7 \times 32$ SRLs are used to implement the 32-bit 32-length shift registers, which store the expanded messages for $X$ and $Y$ alternatingly. With the registers shifting, the expansion function circuit is reused for the alternative messages.

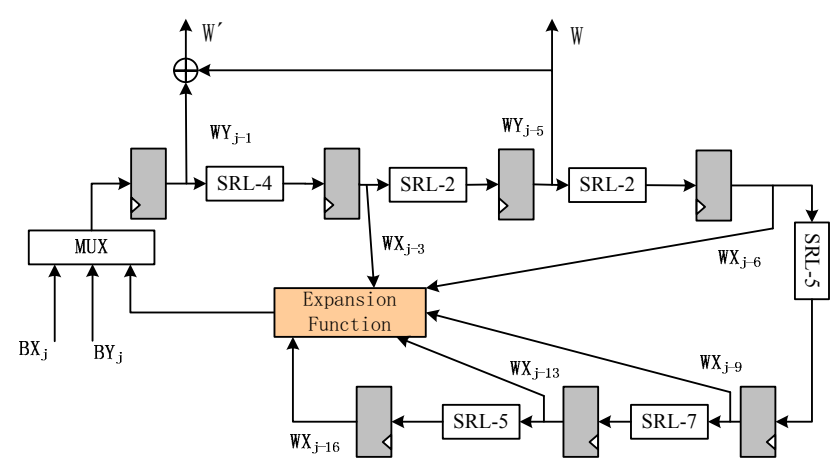

Fig. 7. The SRL-based message expansion structure for two messages 


\section{Hardware Performance Evaluation}

As there is no hardware implementation of SM3 in literature, we provide a standard SM3 implementation as a baseline for our optimized implementations. Honestly, we do not intentionally make it slower or larger. The standard implementation is defined as follows.

- The message padding module is not included. As the message padding is performed once to the end of the message, and has no significant cost when implemented in software. This is consistent with majority of other hash cores.

- The message expansion module is implemented based on the structure as shown in Figure 2, which is a common implementation in other hash algorithms such as SHA-1.

- The iteration process works as follows. Variables $A$ to $H$ are initialized with $I V$ for the first message block. The iteration module is idle in the first four cycles waiting for $W_{0}{ }^{\prime}$. In the iteration, The assignments of $A$ and $E$ are implemented according to Figure 1. At the last iteration round, besides the iteration operation, $A$ to $H$ performs XOR operation with $V_{0}$ to $V_{7}$.

- The intermediate value $V_{0}$ to $V_{7}$ are assigned as follows. For the first message block, $V_{0}$ to $V_{7}$ are also initialized with $I V$. For the subsequent blocks, $V_{0}$ to $V_{7}$ are assigned by $A$ to $H$ at the first iteration round.

Table 1. Hardware Performance of SM3 and SHA-256 implementations

\begin{tabular}{|c|c|c|c|c|c|c|}
\hline Name & Devices & Slices & $\begin{array}{c}\text { Max Freq. } \\
(\mathrm{MHz})\end{array}$ & Bits/cycle & $\begin{array}{c}\text { ThrPut. } \\
(\mathrm{Mbps})\end{array}$ & ThrPut/Slice \\
\hline \hline Standard-SM3 & Virtex-5 & 384 & 214 & 7.53 & 1611 & $\mathbf{4 . 2 0}$ \\
\hline \hline C-SM3 & Virtex-5 & 234 & 215 & 7.53 & 1619 & $\mathbf{6 . 9 2}$ \\
\hline T-SM3 & Virtex-5 & 328 & 362 & 7.53 & 2726 & $\mathbf{8 . 3 1}$ \\
\hline \hline SHA-256 $[2]$ & Virtex-5 & 319 & 221 & 7.76 & 1714 & $\mathbf{5 . 3 7}$ \\
\hline
\end{tabular}

In order to evaluate our SM3 designs, both the compact and the high-throughput designs are implemented on Xilinx Virtex-5 (XC5VLX110T-3) FPGA, and Place and Route by Xilinx ISE 13.2. For simplicity, the compact and high-throughput implementations are named C-SM3 and T-SM3, and the standard one named Standard-SM3. Table 1 shows the comparison results of implementation performance in terms of slices, working frequency, bits processed per cycle and throughput per slice (ThrPut/Slice).

Implementation results indicate that $C$-SM3 only occupies 234 Slices and $P$ $S M 3$ achieves a high throughput of 2.7 Gbps on Virtex-5. In addition, both the two implementations achieve a high ratio of throughput to area.

Compared with Standard-SM3, C-SM3 only occupies $61 \%$ area with no degradation of throughput, resulting in that the Throughput/Slice increases $65 \%$. 
Also, compared with Standard-SM3, T-SM3 improves the throughput by $69 \%$, and also saves $17 \%$ area. When compared with the compact implementation, the high-throughput one also achieves a $68 \%$ throughput improvement with only a $40 \%$ area increase, resulting in that the ratio of throughput to area increases by $20 \%$. The comparison result suggests that our optimization techniques are not only effective for the one-message architecture, but more efficient for the two-message architecture.

When compared with the best SHA-256 implementation [2], our $C$-SM3 occupies $73 \%$ slices, while achieving a $29 \%$ improvement to ThrPut/Slice. This means that our SM3 implementation can occupy much smaller area with a competitive throughput.

\section{Related Work}

Several techniques have been proposed to improve the FPGA implementations of hash algorithms, such as using CSAs, using embedded memories, pipelining, unrolling and rescheduling.

CSA techniques are used to improve the partial additions 34. We also use CSAs in the standard and optimized implementations. The rotation operation $\lll 7$, however, limits further optimization for shortening the critical path.

The usage of embedded memories such as block RAMs to store the required constants can save resources [3 45]. The technique is useless for SM3 implementation, as there are not substantive constants need to be stored.

Pipelining techniques are helpful to achieve higher working frequencies 3 [56]. These technique, however, can hardly improve the maximum frequency in SM3 implementations, due to the rotation operation $\lll 7$ in $S S 1$ and the strong dependency of variables $A$ and $E$. The details have been explained in Section 3.1. Unrolling techniques optimize the data dependency [46/789], while they may significantly increase the required hardware resources .

The pipeline architecture for multiple messages is also useful to achieve a high throughput for multiple independent messages [7810. The main idea of the techniques is reusing the calculation logic to decrease the area and processing multiple messages simultaneously to increase the throughput. Nonetheless, the register resources are duplicated several times. Our optimization techniques provide a good means to minimize the registers in the multiple-message architecture.

Rescheduling and hardware reutilization techniques can improve hardware realizations both in speed and in area [35]. The rescheduling technique can not be applied in SM3 implementation directly, due to the ROL operations $\lll 9$ for $B$ and 19 for $F$ in the iteration of SM3. Instead of the finalization operation within the 64-round iteration in the techniques, our finalization operation is completed before the iteration begins, which allows us to remove many multiplexers for the variables $A$ to $H$. 


\section{Conclusion}

This paper optimizes and evaluates the hardware performance of SM3 hash algorithm on FPGA for the first time. We propose novel techniques to optimize the SM3 implementation, since common optimization techniques are not applicable. Implementation results clearly indicate that our techniques allow a substantial reduction on reconfigurable resources, either in the compact architecture or in the high-throughput architecture. Thus, SM3 with higher efficiency is suitable for hardware implementations, especially for the resource-limited hardware platforms. In fact, our optimization techniques can also be used for the implementations of other hash algorithms, such as SHA-2. We will work on this issue in the next stage.

\section{References}

1. Chinese Commercial Cryptography Administration Office: Sepecification of SM3 Cryptographic Hash Function (2010) (in Chinese), http://www.oscca.gov.cn/UpFile/20101222141857786.pdf

2. HELION: Fast SHA-256 core for xilinx FPGA (2011), http://www.helion.com/

3. Chaves, R., Kuzmanov, G., Sousa, L., Vassiliadis, S.: Improving SHA-2 Hardware Implementations. In: Goubin, L., Matsui, M. (eds.) CHES 2006. LNCS, vol. 4249, pp. 298-310. Springer, Heidelberg (2006)

4. McEvoy, R.P., Crowe, F.M., Murphy, C.C., Marnane, W.P.: Optimisation of the SHA-2 Family of Hash Functions on FPGAs. In: IEEE Computer Society Annual Symposium on Emerging VLSI Technologies and Architectures (ISVLSI), pp. 317$322(2006)$

5. Chaves, R., Kuzmanov, G., Sousa, L., Vassiliadis, S.: Cost-Efficient SHA Hardware Accelerators. IEEE Transactions on Very Large Scale Integration (VLSI) Systems 16(8), 999-1008 (2008)

6. Macchetti, M., Dadda, L.: Quasi-Pipelined Hash Circuits. In: IEEE Symposium on Computer Arithmetic, pp. 222-229 (2005)

7. Michail, H.E., Kakarountas, A.P., Selimis, G.N., Goutis, C.E.: Optimizing SHA-1 Hash Function for High Throughput with a Partial Unrolling Study. In: Paliouras, V., Vounckx, J., Verkest, D. (eds.) PATMOS 2005. LNCS, vol. 3728, pp. 591-600. Springer, Heidelberg (2005)

8. Lee, E.H., Lee, J.H., Park, I.H., Cho, K.R.: Implementation of High-Speed SHA-1 Architecture. IEICE Electronics Express 6(16), 1174-1179 (2009)

9. Sklavos, N., Koufopavlou, O.: Implementation of the SHA-2 Hash Family Standard Using FPGAs. The Journal of Supercomputing 31(3), 227-248 (2005)

10. Kakarountas, A.P., Michail, H., Milidonis, A., Goutis, C.E., Theodoridis, G.: HighSpeed FPGA Implementation of Secure Hash Algorithm for IPSec and VPN Applications. The Journal of Supercomputing 37(2), 179-195 (2006) 